Sādhanā Vol. 40, Part 4, June 2015, pp. 1169-1184. (C) Indian Academy of Sciences

\title{
Evolutionary robotics in two decades: A review
}

\author{
SAMEER GUPTA and EKTA SINGLA* \\ School of Mechanical, Materials and Energy Engineering, IIT Ropar, Rupnagar \\ 140001, India \\ e-mail: sameer.gupta@iitrpr.ac.in; ekta@iitrpr.ac.in
}

MS received 29 August 2014; revised 1 January 2015; accepted 11 February 2015

\begin{abstract}
Evolutionary robotics (ER) has emerged as a fast growing field in the last two decades and has earned the attention of a number of researchers. Principles of biological evolution are applied in the form of evolutionary techniques for solving the complicated problems in the areas of robotic design and control. The diversity and the intensity of this growing field is presented in this paper through the contributions made by several researchers in the categories of robot controller design, robot body design, co-evolution of body and brain and in transforming the evolved robots in physical reality. The paper discusses some of the recent achievements in each of these fields along with some expected applications which are likely to motivate the future research. For the quick reference of the readers, a digest of all the works is presented in the paper, spanning the years and the areas of the research contributions.
\end{abstract}

Keywords. Evolutionary robotics; evolutionary control; robot morphology; bodybrain design.

\section{Introduction}

With the advent of bio-inspired computational techniques (Brooks 1986; Beer \& Gallagher 1992; Haykin 1994; Ram et al 1994), the interests of many roboticists bend towards the utility of these techniques in the complicated designs and control of robots. The population-based evolutionary methods iteratively learn the features of the present scenario, eventually adapt the environment and are expected to evolve out certain features which are not yet present in the initial population. This ability of the evolutionary techniques to generate super-individuals is the base for their popularity. With the work of (Sims 1994a), the feasibility to obtain an evolutionary process to simultaneously design robot morphology as well as robot brain has been introduced. The efficacy of the techniques in handling challenging problems of robot design and control has opened up the field of evolutionary robotics (ER) for further exploration. This paper presents a collection of works contributed in this area, classifies them for their quick representation and discusses the possible directions for future works.

*For correspondence 
Algorithms in evolutionary robotics usually operate on a set of population (solutions), initially selected from random distribution. This set of population is then repeatedly modified according to the fitness function(s), which are formulated to express the objective(s) of a problem. The aspects of non-continuity and multimodality are catered commendably through evolutionary algorithms (EA). By providing the possibility of working through all the possible solutions and selecting the most suitable solution to a problem, EA serves as a strong platform to work upon. Commonly used evolutionary techniques in this field of ER are genetic algorithms and genetic programming. After a brief description of some selected techniques in section 2, a state-of-theart literature review in evolutionary robotics is presented in section 3 . The section categorizes the contributions made in this field into four broad areas - evolutionary robotics control, design using evolutionary computations, co-evolution of robot body and brain and the works related to the evolution of robot design and its prototype. For a quick referral by the readers, the authors have worked upon a digest of the previous works, spanned by the categories and the published years. Each part of the digest is presented through a figure. Future challenges related to evolutionary robotics are discussed in section 4 followed by a summary of the discussion.

\section{Computational tools}

Some of the techniques like genetic algorithm have been used to a great extent in the field of evolutionary robotics. Most of the works presented in recent years are mainly based upon the methodologies tailored out of the basic genetic algorithm. Neural networks is another majorly used tool in the evolution of the controllers. There have been other techniques applied for the field e.g. generative representation, NeuroEvolution of Augmenting Topologies (NEAT). A few of these tools have been briefly described in this section before discussing their utilization in the design and control problems.

\subsection{Genetic algorithm}

Genetic algorithm (GA) belongs to a large class of evolutionary algorithms which generate solutions to optimization problems using techniques inspired by natural evolution (Holland 1975; Goldberg \& Holland 1988; Goldberg 1989; Michalewicz 1996; Deb 2012; Pratihar 2013). In genetic algorithm a set of population, termed as candidate-solutions of an optimization problem, is evolved over and over to direct itself towards optimal solution(s). The candidates are initially encoded in specified format depending on real or binary genetic representation. Considering the case of binary encoding, the properties of a candidate solution are represented through a corresponding string consisting of 1 or 0 at different locations.

There are basically three operators - reproduction, crossover and mutation - that are used for evolving the population to next generations, as shown in figure 1. A fitness function is employed to measure the fitness of each candidate. Reproduction operator helps in the selection of best candidates in each generation using probability based techniques. After the selection of best candidates, a mating pool is formed. Crossover operator uses this mating pool and randomly selects a site at which crossover operation has to be carried out as shown in figure $2 \mathrm{a}$. At the end of the crossover operator, mutation operator is used which induces some specific features in the offspring candidates which are not present in their parent candidates. This operator flips the value of binary digit in a string as shown in figure $2 \mathrm{~b}$, from $1 \rightarrow 0$ or $0 \rightarrow 1$, at a location selected with a small probability. This may help the population in coming out of a local minima. 


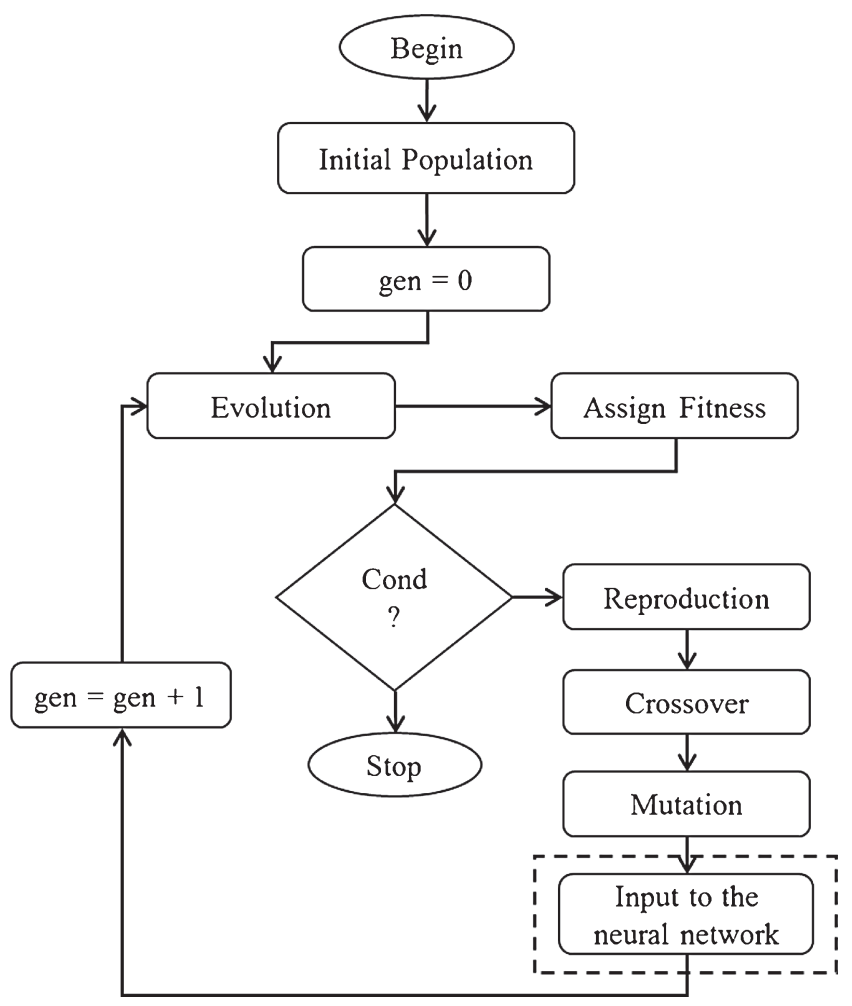

Figure 1. Flowchart of genetic algorithm.

\subsection{Neural networks}

Neural Networks (NN) is an information processing model that is inspired by the working of biological nervous system and process information similar to the brain (Holland 1975, 1992; Haykin 1994; Pratihar 2013). The key element of this model is the structure of its information processing system which is basically composed of a large number of highly interconnected processing elements - termed as neurons - through synapse working in unison to solve specific problems. Neural networks consists of a net of neurons (McCulloch \& Pitts 1943), similar to a human being, learn by experience. A neural network is normally used to configure parameters, through a learning process, for a specific purpose such as robot's control.

In neural network, there are three important layers as shown in figure 3, namely input layer, hidden layer and output layer. The input layer consists of sensors which fetch the feedback signals from the environment and send them to the hidden layer. The elements of the hidden layer may also be connected to each other in case of recurrent neural network. Now, hidden layer processes the signal and finally sends the output signal to the output layer. Based on the

$$
\begin{aligned}
& 1010|01011 \rightarrow 1010| 10110 \quad 101010110 \rightarrow 100010110 \\
& 1000 \$ 10110 \rightarrow 1000 \mid 01011 \quad 100001011 \rightarrow 100001111
\end{aligned}
$$

(a)

Figure 2. (a) Crossover operator, (b) Mutation operator. 


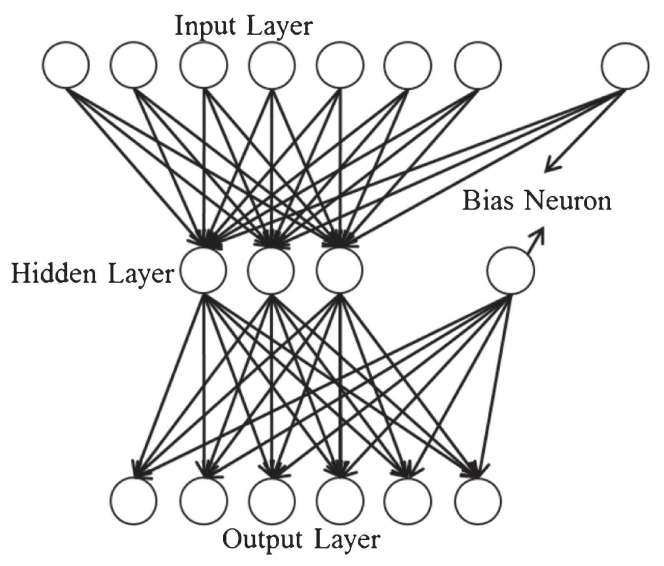

Figure 3. Neural network.

resulting output, the actuators perform their actions. Flowchart in figure 1 represents the training scenario of neural networks using genetic algorithm, where dotted box represents the block of neural networks. This dotted box in figure 1 can be replaced by figure 3 to represent the process of training to neural network.

\subsection{NeuroEvolution of augmenting topologies}

NeuroEvolution of Augmenting Topologies (NEAT), developed by Ken Stanley (Stanley \& Miikkulainen 2002) in 2002, is a genetic algorithm used for evolving artificial neural networks. It alters both the weighing parameters and the structure of the network to find a balance between the fitness of evolved solutions and their diversity. It applies three key steps: tracking genes with history markers to allow crossover among topologies, applying speciation (the evolution of species) to preserve innovations and developing topologies incrementally from simple initial structures. NEAT offers a solution to the problem of competing conventions in a population of diverse topologies. NEAT also demonstrates that a meaningful metric for comparing and clustering similar networks can easily be derived from the availability of historical information in the population and costly topological analysis is not necessary to speciate or mate networks. NEAT (Stanley \& Miikkulainen 2002) starts with a population of simple neural networks and then adds complexity over generations by adding new nodes and connections through mutations. By evolving networks in this way, the topology of the network does not need to be known a priori. NEAT searches through increasingly complex networks to find a suitable level of complexity and tends to find a solution network close to the minimal necessary size. It had been confirmed from the study (Stanley \& Miikkulainen 2002) that starting with a population of minimal topologies is advantageous.

\subsection{Generative representation}

Generative representation is a class of representations in which the encoded data structure of a design can be reused recursively in creating a design. This type of representation allows the robot's morphology to develop from a basic 'seed' and a set of context-free development rules. As an example, considering two rules - ' $\mathrm{B} \rightarrow \mathrm{A}$ ' and ' $\mathrm{A} \rightarrow \mathrm{ABA}$ ' - if 
we start with a seed value 'A' and apply these two rules recursively, the value of the seed will evolve as follows: $\mathrm{A} \rightarrow \mathrm{ABA} \rightarrow$ ABAAABA $\rightarrow$ ABAAABAABAABAAABA $\rightarrow$ ABAAABAABAABAAABAABAAABAABAAABAABAABAAABA and so on. This type of generative representation scheme was first proposed by (Lindenmayer 1968) and was known after his name as Lindenmayer system (L-System). Thus, generative representation is an Lsystem program that after compilation produces a sequence of build commands known as assembly procedure, and a constructor then executes the assembly procedure to generate both robot morphology and robot controller. A comprehensive analysis of generative representation system along with various applications like automated design of modular robots has been carried out so far (Wiener-Ehrlich et al 1980; Hornby \& Pollack 2002; Hornby et al 2003).

\section{Previous works and achievements}

Pioneered by the works of Sims (1994a), the field of ER has evolved out in three major directions - evolutionary robotics control, evolutionary robotics design and co-evolution of morphology and brain. Along with these three directions, another related upcoming field deals with the evolution of robot prototypes.

\subsection{Evolutionary robotics control}

A number of researchers have worked for evolving robot brains using artificial evolution. In general, neural networks are being employed to teach a robot about tasks in various exemplary situations and then the robot is expected to take the decisions in unknown situations, depending upon the induced knowledge. In neural network, some weights are used which determine the strength of a signal directed from one layer of neurons to the next one. These weights help in tuning the controller according to different conditions.

Evolutionary computational techniques, such as genetic algorithm, are applied to the neural controller for determining the optimized weights of the controller. Beer \& Gallagher (1992) had applied EA in evolving an adaptive controller for a six-legged insect-like robot. For a changed environment, the controller parameters like time constants, thresholds and connection weights of neural network are evolved. Another work by (Ram et al 1994) utilized the strengths of EA in evolving a collision free path for wheeled robots using genetic algorithms. The navigational problem is divided into basic behaviors - move-to-goal, avoid-static-obstacles, stay-on path, etc. These behaviors are implemented in genetic algorithm to evolve collision free paths. Similar work of collision avoidance in a navigation problem is presented by Grefenstette \& Schultz (1995) for a Nomad 200 mobile robot in the presence of some moving obstacles.

During 1990s, most of the researchers working in controller designs had shown interest in evolutionary techniques for automatic evolution of the controllers. Refer the digest in figure 4 for year wise representation of several contributions. Meeden (1996) worked on the controller of a 4-wheeled robot considering - avoid contact from the walls using a light source as and when required - as the fitness function in the work. During the same time, Baluja (1996) developed an EA-based neural controller using Population-Based-Incremental-Learning (PBIL). Performance of the algorithm has been tested for steering control of a mobile robot. A two-stage controller for two wheeled soccer-playing robots using genetic algorithm has been developed by Jeong \& Lee (1997). In the first stage, the position of the ball, the opponents, the partners and the goal along with the actions like move, dribble or kick are considered. Then, in the second stage optimal onoff signals are considered. In many of the works presented in the late 1990s, the development 


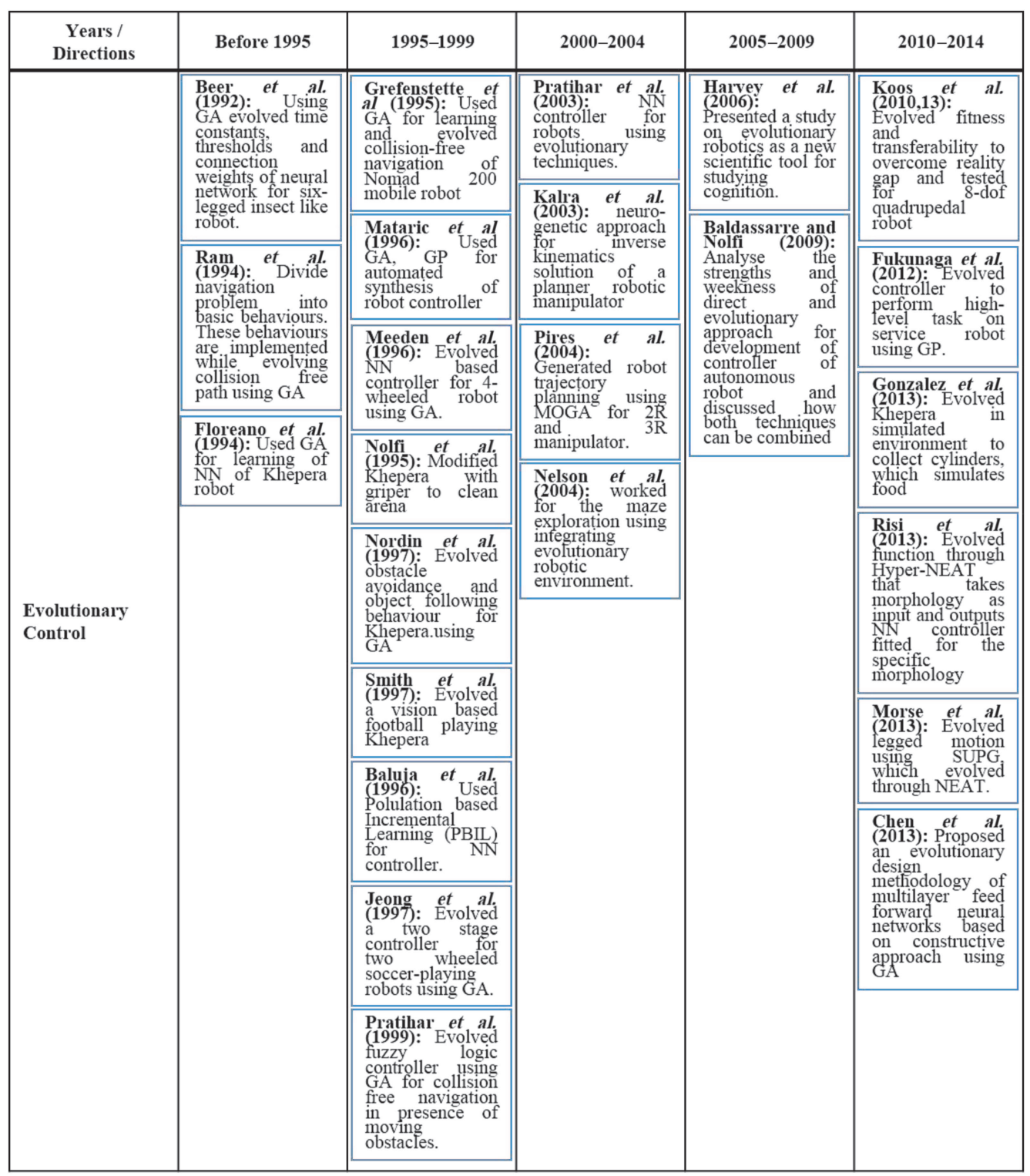

Figure 4. Evolutionary control.

of various features of controller for Khepera, a miniature mobile robot, had been studied for different situations (Floreano et al 1994; Nolfi \& Parisi 1995; Nordin \& Banzhaf 1997; Smith 1997). Very recently, the behavior evolution of Khepera in motivated environment is presented by Montes-Gonzalez \& Contreras (2013), where the task is to collect and consume food cylinders based upon obesity and anorexia. In the era 2001-2009, similar strategies have been successfully implemented for the controller designs of various robotic mechanisms (Pratihar et al 1999; Kalra \& Prakash 2003; Nelson et al 2004; Pires et al 2004; Harvey et al 2005). A review paper by (Pratihar 2003) presents the works on evolutionary control. 
Recently, (Koos et al 2010, 2013) worked upon the reality gap that signifies the inefficiency of the simulation once transferred onto the real system. This issue prevents evolutionary robotics applications to work in real-world. The authors introduced a simulation-to-reality disparity (STR disparity) measure. The higher the STR disparity, the worse the transferability from the simulation to a real-time system is. Two objectives - fitness and transferability - had been considered for the first time for the evolutionary control applications. The work had been demonstrated through the controller development of a walking 8-DOF quadruped robot and for the navigation task of an e-puck robot. Some other recent works presented in the area of evolutionary robotics control are discussed in (Piltan et al 2011a, b, 2013; Fukunaga et al 2012; Saad et al 2012; Chen et al 2013; Černỳ \& Kubalík 2013; Klaus et al 2013; Shenfield \& Fleming 2014). The work presented so far normally involves a specific morphology for which a controller is evolved. Some of the recent developments in the area of co-evolution of morphology and controller have been discussed in a separate section. However, a recently presented study (Risi \& Stanley 2013) had added a new dimension in this field of evolutionary control, through a method called Hypercubebased NeuroEvolution of Augmenting Topologies (Hyper-NEAT). The strategy emphasizes on evolving a function which can present a controller for a given morphology, instead of training a controller for a particular quadruped morphology. Such a relationship is expected to help in the controller designs for a diversity of different morphologies. The work has been illustrated through three different robot morphologies which differ in the length of their legs. Some more work in this direction has been presented by (Morse et al 2013), addressing the challenge of maintaining long-term stability.

The field of evolutionary robotics, for a long time, has remained inclined towards the challenging tasks of robot control. The research works in this area has certainly covered various milestones and still the field is open for further intricacies, related to complex mechanisms, dynamic environments, cluttered workspaces, etc. More importantly, the controllers are required to be flexible to the environment and/or to the changes in the mechanism.

\subsection{Evolutionary robotics design}

Evolutionary computational techniques have been successfully applied by many researchers in robot morphology design problems. Figure 5 presents a year wise collection of some of the published works in this direction. The challenging problem of selecting a specific morphology for a given set of tasks has remained the focus of the research.

Chocron \& Bidaud (1997) proposed the task-based design of modular manipulators using a two-level genetic algorithm, where the upper level is used to evolve the robotic topology and the lower level bounds the search for a subtask configuration. The solution of the upper level is used as an input to the lower level genetic algorithm. During the same time, (Chung et al 1997) worked for the optimal determination of link lengths of modular manipulators by using genetic algorithm. The evolutionary approach had also been applied to evolve the different 2D/3D morphologies using LEGO (Funes \& Pollack 1998, 1999) components. Required morphologies are selected based upon artificial selection algorithms using LEGO components.

In spite of selecting morphologies only from given set of modules or components, another generative approach is presented by (Hornby et al 2001a, 2003; Hornby \& Pollack 2001) which is composed of grammar-like rules for generating physical systems. Generative representation is an L-System program that produces a sequence of build commands to work as an assembly procedure. A generative design specification can reuse components, giving it the ability to create complex modules from simpler ones. The work demonstrated a generative system for physical modular 2D locomotive robots. 


\begin{tabular}{|c|c|c|c|c|c|}
\hline $\begin{array}{c}\text { Years / } \\
\text { Directions }\end{array}$ & Before 1995 & 1995-1999 & 2000-2004 & 2005-2009 & 2010-2014 \\
\hline \multirow{8}{*}{$\begin{array}{l}\text { Evolutionary } \\
\text { Design }\end{array}$} & & $\begin{array}{l}\text { Chedmail et al } \\
\text { (1996): used GA } \\
\text { to open lopp } \\
\text { robot mot for } \\
\text { morphology and } \\
\text { to follow a given }\end{array}$ & \multirow{4}{*}{ 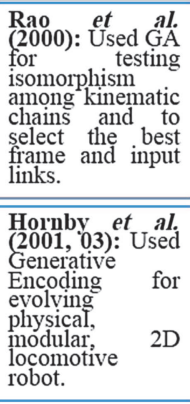 } & $\begin{array}{l}\text { Zykov et al. } \\
\text { (2007): } \\
\text { Demonstrates } \\
\text { robots capable of } \\
\text { autonomous self- } \\
\text { reproduction }\end{array}$ & \multirow{2}{*}{$\begin{array}{l}\text { Bongard et al } \\
\text { (2013): Evolved } \\
\text { morphology of } \\
\text { robots in form of } \\
\text { legs and in later } \\
\text { stage algorithm } \\
\text { developed gait } \\
\text { for the tegs } \\
\text { finally evolved }\end{array}$} \\
\hline & & obstacles & & \multirow{2}{*}{$\begin{array}{l}\text { Rout et al } \\
\text { (2009): } \\
\text { differential Used } \\
\text { evolutionary } \\
\text { optimization for } \\
\text { 2-dof RR planar } \\
\text { and } \\
\text { SCARA 4-dof } \\
\text { manipulators. }\end{array}$} & \\
\hline & & $\begin{array}{l}\text { (1997): Proposed } \\
\text { two level GA } \\
\text { for task-based } \\
\text { design of 3D } \\
\text { modular of } \\
\text { manipulator. }\end{array}$ & & & \multirow{2}{*}{$\begin{array}{l}\text { Rubrecht et al. } \\
\text { (2011): Evolved } \\
\text { a manipulator } \\
\text { working in a a } \\
\text { highly } \\
\text { constrained } \\
\text { workspace of the } \\
\text { maintenance of } \\
\text { TBM in hostile } \\
\text { conditions using } \\
\text { GA }\end{array}$} \\
\hline & & \multirow{2}{*}{$\begin{array}{l}\text { Chung et al. } \\
\text { (1997): } \\
\text { length of takk- } \\
\text { based modular } \\
\text { manipulator was } \\
\text { determined } \\
\text { through GA after } \\
\text { determining } \\
\text { necessary } \\
\text { configuration }\end{array}$} & & & \\
\hline & & & & & \multirow{2}{*}{$\begin{array}{l}\text { Tolley et al. } \\
\text { (201): } \\
\text { Presented an } \\
\text { automated anded } \\
\text { approach } \\
\text { stochastic to } \\
\text { modular robotics } \\
\text { that can evolve a } \\
\text { structure and } \\
\text { assembly plan to } \\
\text { achieve a target } \\
\text { function. }\end{array}$} \\
\hline & & \multirow[t]{3}{*}{$\begin{array}{l}\text { Funnes et al } \\
\text { (1998, 99). Used } \\
\text { GA to evolve the } \\
\text { different 2D/3D } \\
\text { morphologies } \\
\text { using LEGO }\end{array}$} & & & \\
\hline & & & & & 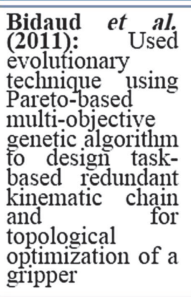 \\
\hline & & & & & 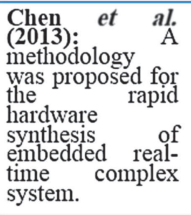 \\
\hline
\end{tabular}

Figure 5. Evolutionary design.

Further works on the similar pattern, for spatial robots, have been carried out by many researchers during last decade. A demonstration of a large space of possible robots, capable of autonomous self-reproduction, had been carried out by Zykov et al (2007). These robots are composed of actuated modules equipped with electromagnets to selectively control the morphology of the robotic assembly. Both automatically generated and manually design morphologies have been presented in the paper. Rout \& Mittal (2010) proposed a modification in the differential evolution optimization technique to incorporate the effect of noises in the optimization process and obtain the optimal design of manipulators. Optimal parameters of 2-DOF RR planar manipulator and of 4-DOF SCARA manipulator have been designed and the results have been compared using genetic algorithm with similar modifications. It had been found that less computational effort is required in the differential evolutionary optimization. An automated approach to stochastic modular robotics had been presented (Tolley et al 2011) that can evolve a structure to achieve a target function. 
In recent works, evolutionary methods have been applied for the design of more complicated robotic mechanisms. It can be noted that in all these works, presumptions of a specific morphology for a robot are taken a priori. In one of such contributions (Bongard 2011), a mechanism is evolved in the form of legs and later a gait is developed for the evolved legs. The authors have worked in the optimizational synthesis of redundant kinematic chains (Singla et al 2010) for working in cluttered environments. A constrained optimization problem was formulated for reaching at all given task locations while avoiding any collision of the manipulator. Later, a serial robot had been designed for the maintenance of a (Tunnel Boring Machine) TBM, for its maintenance in hostile conditions (Rubrecht et al 2011). Multi-objective Genetic Algorithm had been applied to handle the highly constrained environments and to preserve a good exploration space. The author's team had also explored the application of Variational principle in the optimal path planning of serial manipulators, working in cluttered workspaces (Shukla et al 2013). The approach involves formulating the path planning problem as a constrained minimization of a functional representing the total joint movement over the complete path. The challenges related to a large number of degrees of freedom of redundant manipulators, to collisions related to cluttered spatial workspaces and to other aspects of discrete design variables and multiple objectives have been worked upon through the evolutionary approach of genetic algorithms.

More such applications of evolutionary techniques for complicated mechanisms working in cluttered environments are worth paying attention. Further investigation is also expected for the evolutionary designs of autonomous manipulators for any given environment.

\subsection{Co-evolution of morphology and brain}

Introduced by the work of (Sims 1994a, b), the field of co-evolving morphologies and control has received several contributions thereafter. The work shows that a number of aspects related to one physical life-form can be connected together to obtain an optimized life-form. In this direction, (Lee et al 1996; Lund et al 1997) developed a hybrid approach to evolve both the controllers and the robot bodies simultaneously, for behavior-specified tasks, using both genetic algorithm and genetic programming.

Chocron \& Bidaud (1999a, b) presented an integrated dynamic simulation for locomotive robotic systems. Types of wheels, links and joints, along with their best suitable locations are represented in genotype form. The locomotion is then evolved along with its controller using genetic algorithm. Chocron et al (2005) recently presented a global design approach for selfreconfigurable locomotion systems based on dynamic simulations and artificial evolution. Main objective of this approach is to obtain fully integrated robotic solutions in terms of morphology (topology and kinematics) and control (architecture and command). Both topology and control have been co-evolved through genetic algorithm. Study has been presented on different examples of modular robotic systems like rover and snakes. Similar strategies may be applied for co-evolving some complicated manipulators to be worked in cluttered environments of industries and/or for specific environments arises due to calamities. A platform can be developed for evolving the complete mechanism for a given environment along with its controller.

Inspired by biological life forms, Lipson and Pollack (Lipson \& Pollack 2000; Pollack \& Lipson 2000) presented the evolution of articulated robots using vertices, bars, neurons and actuators through evolutionary processes. Fitness function to evolve the robots is taken as the distance moved by the center of mass of a robot in fixed number of simulation cycles. The physical prototypes of simulated robots had been prepared and compared with the simulated ones. Similar group of researchers had also worked for the construction and control of electromechanical systems and for locomotive creatures (Pollack et al 2000; Hornby et al 2001b). 
The three generations of robots developed by this team, namely, LegoBot, Genetically Organized Lifelike Electro-Mechanics (GOLEM) and Modularity Generative Design (Tinkerbots) have been discussed in Pollack et al (2001).

A stable bipedal locomotion using coupled evolution of morphology and controller for a 5-linked biped robot has been achieved by Paul \& Bongard (2001). A study of effects of microscopic, midrange and macroscopic changes in mass distribution of the bipedal robots has been performed. It has been illustrated that the controller and the design can be evolved simultaneously in a single step. Further, the researchers worked on a method for isolating the effect of morphological changes on the evolved behavior of quadupedal and hexapedal body plans (Pfeifer 2002; Bongard \& Lipson 2005). A co-evolutionary algorithm for inferring the topology and parameters of a wide range of hidden non-linear systems with a minimum of experimentation on the target system has also been presented. A review on works done by Lipson and Pollack's team in the field of evolutionary robotics is comprised in (Lipson 2005). Bongard (2013) had also presented a thorough review on evolutionary robotics.

A chart is presented in figure 6 to sum-up the research presented in last two decades in this direction of co-evolution of robot morphology and control. It is worth mentioning here that not much connectivity has been noticed in the works presented in this direction. The researchers have either investigated the applicability of evolutionary approaches for gait development and/or motion planning of some specific locomotive systems, or the work has been presented from algorithmic perspectives. The threads need to be woven together for possible outcomes of coevolving several kinds of mechanisms with various levels of complexity in their controllers. The research direction possesses strength of shifting the work direction of controllers design for complicated mechanisms to just focus on desired mechanisms which can be co-evolved along with their controllers.

\subsection{Physically prototyped}

A few researchers tried to build the prototype using 3D printing machines and compared the results of evolved simulated robots to the prototyped ones. Figure 7 presents the upcoming research works done with real creature's development as an inherent part of evolutionary process.

In a work presented by Pollack \& Lipson (2000), the physics of robots is evolved using evolutionary processes and then the corresponding physical model is prototyped using 3D printing machine to compare the performance (Hornby \& Pollack 2001). This strong feature in the complete evolutionary process signifies the ability of a system to go from design to manufacturing. A pathway had been demonstrated for the first time that allows transfer of virtual diversity into reality (Lipson \& Pollack 2006). The approach uses only elementary building blocks in both the design and embodiment. A set of preliminary experiments, evolving electro-mechanical systems, are worked upon - first in simulation and then in reality. Using 3D solid printing, these creatures then replicate automatically into reality where they reproduce the performance of their virtual ancestors.

Recently, a novel concept of evolution of things has been introduced (Eiben et al 2012) and implemented for various applications, e.g. robot companion, environment friendly organisms and evolutionary 3D printing. A 3D printing machine, known as EvoFab, has been developed (Rieffel \& Sayles 2010; Kuehn 2012) to fabricate the robots evolved through evolutionary processes so as to cover up the gap between simulation and reality. However, the work is found in one of its kind. Further investigations are required for analyzing the applicability of the proposed concepts for realistic works. Besides, similar works can be taken ahead for co-evolving design and manufacturing methodology and/or the assembly plan, simultaneously. 


\begin{tabular}{|c|c|c|c|c|c|}
\hline $\begin{array}{c}\text { Years / } \\
\text { Directions }\end{array}$ & Before 1995 & 1995-2000 & 2001-2004 & 2005-2009 & 2010-2014 \\
\hline \multirow{14}{*}{$\begin{array}{l}\text { Evolutionary } \\
\text { Morphology and } \\
\text { Control }\end{array}$} & \multirow{2}{*}{$\begin{array}{l}\text { Sims et al. } \\
\text { (1994): } \\
\text { Pioneering the } \\
\text { concept } \\
\text { combining both } \\
\text { morphology and } \\
\text { control using } \\
\text { evolutionary } \\
\text { approach }\end{array}$} & \multirow{2}{*}{$\begin{array}{l}\text { Lee et al. } \\
\text { (1996): Combine } \\
\text { morphology and } \\
\text { control } \\
\text { features for } \\
\text { walking, like } \\
\text { jumping, } \\
\text { swimming and } \\
\text { following using } \\
\text { EA. }\end{array}$} & \multirow{3}{*}{$\begin{array}{l}\text { Paul et all } \\
\text { (2001): } \\
\text { GA asing } \\
\text { achieved stable } \\
\text { bipedal } \\
\text { locomotion using } \\
\text { coupled } \\
\text { evolution of of } \\
\text { morphology and } \\
\text { control on a 5- } \\
\text { link biped robot }\end{array}$} & \multirow{4}{*}{$\begin{array}{l}\text { Chocron et al } \\
\text { (2005): Global } \\
\text { design approach } \\
\text { for } \\
\text { reconfigurable } \\
\text { locomotion } \\
\text { systems based on } \\
\text { evaluation by } \\
\text { dynamic } \\
\text { simulation and } \\
\text { optimization by } \\
\text { artificial } \\
\text { evolution }\end{array}$} & \multirow{5}{*}{$\begin{array}{l}\text { Bongard et al. } \\
\text { (2013): } \\
\text { Presented } \\
\text { review a } \\
\text { evolutionary } \\
\text { robotics }\end{array}$} \\
\hline & & & & & \\
\hline & & Lund et al & & & \\
\hline & & (1997): Include & Hornby et al. & & \\
\hline & & $\begin{array}{l}\text { plan in the } \\
\text { genotype and } \\
\text { co-evolve the } \\
\text { task-fulfilling }\end{array}$ & $\begin{array}{l}\text { P0L-system for } \\
\text { generatively } \\
\text { creating both } \\
\text { creature }\end{array}$ & \multirow{3}{*}{$\begin{array}{l}\text { Lipson et al. } \\
\text { (2005): } \\
\text { Presented a } \\
\text { review of work } \\
\text { done by his team } \\
\text { in the field of } \\
\text { evolutionary } \\
\text { robotics }\end{array}$} & \\
\hline & & $\begin{array}{l}\text { behaviour and } \\
\text { body plan using } \\
\text { GA }\end{array}$ & $\begin{array}{l}\text { morphology and } \\
\text { neural controller }\end{array}$ & & \\
\hline & & Chocron et al & \multirow{2}{*}{$\begin{array}{l}\text { Pollack et al. } \\
\text { (2001): } \\
\text { Presented review } \\
\text { on LegoBot } \\
\text { GOLEM and } \\
\text { Tinkerbots als }\end{array}$} & & \\
\hline & & $\begin{array}{l}\text { (1999): Used GA } \\
\text { for integrating } \\
\text { dynamic } \\
\text { simulation of } \\
\text { global robotic }\end{array}$ & & \multirow{2}{*}{$\begin{array}{l}\text { Paul et al } \\
\text { (2006): Used GA } \\
\text { to obtain periodic } \\
\text { gait controllers } \\
\text { for tensegrity } \\
\text { robots }\end{array}$} & \\
\hline & & system for & \multirow{3}{*}{$\begin{array}{l}\text { Bongard et al: } \\
\text { (2002, } \\
\text { Presented the } \\
\text { method } \\
\text { isolating for } \\
\text { effect the } \\
\text { morphological of } \\
\text { changes on the } \\
\text { evolved } \\
\text { behaviour of } \\
\text { quadupedal and } \\
\text { hexapedal body } \\
\text { plan } \\
\text { locomotion for } \\
\text { GA }\end{array}$} & & \\
\hline & & $\begin{array}{l}\text { environment for } \\
\text { various tasks }\end{array}$ & & & \\
\hline & & $\begin{array}{l}\text { Lipson et al } \\
(2000) \text { Evolved } \\
\text { the articulated } \\
\text { robots inspired } \\
\text { by biological life } \\
\text { form. The robots } \\
\text { were evolved } \\
\text { from vertices } \\
\text { bars, neurons and } \\
\text { actuators by the } \\
\text { use }\end{array}$ & & & \\
\hline & & $\begin{array}{l}\text { evolutionary } \\
\text { strategies. }\end{array}$ & \multirow{2}{*}{$\begin{array}{l}\text { Stanley et al. } \\
(2004) ! \\
\text { competitive } \\
\text { coevolution of } \\
\text { robot neural } \\
\text { controller and } \\
\text { location } \\
\text { sensors of } \\
\text { motors and } \\
\text { NEAT. through } \\
\text { NEAT. }\end{array}$} & & \\
\hline & & $\begin{array}{l}\text { Pollack et al. } \\
\text { (2000): Used GA } \\
\text { for evolution and } \\
\text { construction of } \\
\text { electromechanica } \\
\text { l systems. }\end{array}$ & & & \\
\hline & & $\begin{array}{l}\text { Pollack et al. } \\
(2000): \text { Used GA } \\
\text { for evolution and } \\
\text { construction of } \\
\text { electro- } \\
\text { mechanical } \\
\text { systems. }\end{array}$ & & & \\
\hline
\end{tabular}

Figure 6. Co-evolution of morphology and control.

\section{Motivation for future research}

Most of the studies presented in the area of evolutionary robotics consider both the evolution through generations and the learning of individual entities. Although evolution and learning are two distinct forms of changes, they have strong influence on each other. It has been noted that performance of learning increases when it is combined with evolutionary techniques. Thus, the interaction between learning and evolution and their effects on each other is likely to be studied for the enhancement of the adaptive power of evolutionary processes.

In the area of evolutionary controller design, further investigation is needed for workability and efficacy analyses of flexible neural controllers. To avoid efforts required for morphologyspecific controllers and to independently work upon morphologies with least botheration about 


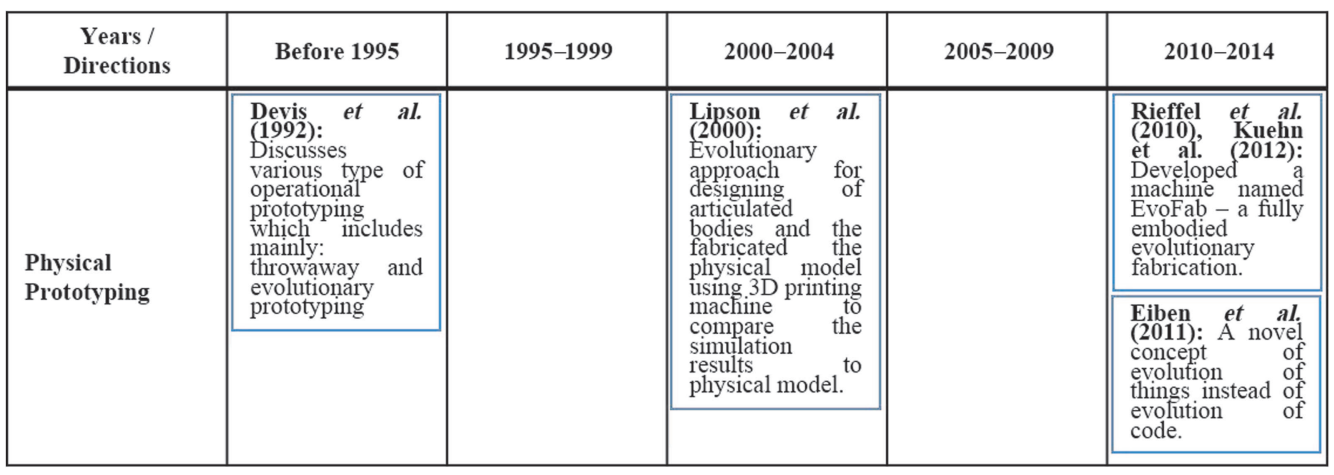

Figure 7. Physical prototyping of evolved robots.

the corresponding controller design, are the points of motivation for pursuing further research in this direction.

Adaptive controllers design strategies are expected to serve a major role in development of modular robotic mechanisms. Use of homogenous or heterogeneous modules, in any number, to develop a robotic mechanism for fulfilling certain task requirements is an extremely challenging problem, without utilization of adaptive controllers.

Apart from the co-evolution of morphology and the corresponding controller, further research would help in exploring the possibilities for co-evolution of desired robotic structure and its assembly plan. With some recent works presented for the co-evolution of a robotic arm and its motion planning, possibility of providing a plan for parts fabrication and assembly steps do exist and it needs further exploration and discussions. More complexity in robotic mechanisms with respect to extra degrees of freedom, working on legs and arms together and/or evolution of co-operative robots are some of the areas worth exploration.

With the tremendously increasing applications of robotic mechanisms, the trend of customized robots - which are designed and developed based on the specific task requirements - is rapidly increasing. Even with a large group of researchers working on general design strategies for various kinds of task-based robots, the bottleneck remains with the transformation of the complicated designs into real mechanisms. Co-evolution of design and fabrication parameters is expected to change the complete format of developing such smart machines. The latest innovations and upcoming possibilities in rapid prototyping techniques, automatic micro machining methodologies certainly are expected to play a strong role in co-evolution.

Emotions do play an important role, along with intelligence, in decision-making process of a human being. The aspects of artificial emotions have already been connected, in some studies, with the intelligence aspects like perception, planning and control. It will be better to implement in the evolutionary processes these studies on interactions of different aspects of a robotic system to evolve its appropriate cognitive architecture.

\section{Summary}

The paper presents a review on the contributions made by several researchers in the implementation of evolutionary techniques in robot controller design, robot body design, co-evolution of body and brain and in transforming the evolved robots in physical reality. The field of evolutionary robotics has broadened the limits on the transformation of a new robotic idea into reality. The 
tasks related to design, development and control of robotic mechanisms have always remained challenging due to the involvement of complicated dynamics and unknown and/or cluttered environments. Evolutionary techniques have served as a strong platform for evolving the designs, controllers and even their combinations to cater the requirements. Through the discussion on various dimensions of this field of evolutionary robotics, the paper highlights the efficacy of evolution. To support the discussion and analysis presented in the paper, a research digest has been compiled and included in the paper in four separate parts.

\section{References}

Baluja S 1996 Evolution of an artificial neural network based autonomous land vehicle controller. Syst. Man Cybern. B Cybern., IEEE Trans. 26(3): 450-463

Beer R D and Gallagher J C 1992 Evolving dynamical neural networks for adaptive behavior. Adaptive Behav. 1(1): 91-122

Bongard J 2011 Morphological change in machines accelerates the evolution of robust behavior. Proc. Natl. Acad. Sci. 108(4): 1234-1239

Bongard J C 2013 Evolutionary robotics. Communications of the ACM 56(8): 74-83

Bongard J C and Lipson H 2005 Nonlinear system identification using coevolution of models and tests. Evolut. Comput., IEEE Trans. 9(4): 361-384

Brooks R A 1986 A robust layered control system for a mobile robot. Robot. Autom., IEEE J. 2(1): 14-23

Černỳ J and Kubalík J 2013 Co-evolutionary approach to design of robotic gait. Springer

Chen C-H, Yao T-K, Kuo C-M and Chen C-Y 2013 Evolutionary design of constructive multilayer feedforward neural network. J. Vibr. Control 19(16): 2413-2420

Chocron O and Bidaud P 1997 Genetic design of 3d modular manipulators. In: Robotics and Automation, 1997. Proceeding, 1997 IEEE International Conference on, vol. 1, pp. 223-228

Chocron O and Bidaud P 1999a Evolutionary algorithm for global design of locomotion systems 3 : 15731578

Chocron O and Bidaud P 1999b Evolving walking robots for global task based design. In: Evolutionary Computation, 1999. CEC 99. Proceedings of the 1999 Congress on, vol. 1

Chocron O, Brener N, Bidaud P and Amar F B 2005 Evolutionary synthesis of structure and control for locomotion systems. In: Climbing and Walking Robots, pp. 879-888. Springer

Chung W K, Han J, Youm Y and Kim S 1997 Task based design of modular robot manipulator using efficient genetic algorithm. In: Robotics and Automation, 1997. Proceedings, 1997 IEEE International Conference on, vol. 1, pp. 507-512

Deb K 2012 Optimization for engineering design: Algorithms and examples. PHI Learning Pvt. Ltd

Eiben A, Kernbach S and Haasdijk E 2012 Embodied artificial evolution. Evolut. Intell. 5(4): 261-272

Floreano D, Mondada F and et al 1994 Automatic creation of an autonomous agent: Genetic evolution of a neural-network driven robot. From animals to animats 3: 421-430

Fukunaga A, Hiruma H, Komiya K and Iba H 2012 Evolving controllers for high-level applications on a service robot: A case study with exhibition visitor flow control. Genetic Programm. Evolvable Mach. 13(2): 239-263

Funes P and Pollack J 1998 Evolutionary body building: Adaptive physical designs for robots. Artif. Life 4(4): 337-357

Funes P and Pollack J 1999 Computer evolution of buildable objects. Evolut. Des. Comput. 1: 387-403

Goldberg D E 1989 Genetic algorithms in search. Addison-Wesley Professional

Goldberg D E and Holland J H 1988 Genetic algorithms and machine learning. Mach. Learn. 3(2): 95-99

Grefenstette J and Schultz A 1995 An evolutionary approach to learning in robots. Technical report, DTIC Document

Harvey I, Di Paolo E, Wood R, Quinn M, Tuci E and Iridia E T 2005 Evolutionary robotics: A new scientific tool for studying cognition. Artif. Life 11(1-2): 79-98 
Haykin S 1994 Neural networks: a comprehensive foundation. Prentice Hall PTR

Holland J H 1975 Adaptation in natural and artificial systems. Ann Arbor, MI: The University of Michigan Press

Holland J H 1992 Adaptation in natural and artificial systems: An introductory analysis with applications to biology, control, and artificial

Hornby G S and Pollack J B 2001 The advantages of generative grammatical encodings for physical design. In: Evolutionary Computation, 2001. Proceedings of the 2001 Congress on, vol. 1, pp. 600-607

Hornby G S and Pollack J B 2002 Creating high-level components with a generative representation for body-brain evolution. Artif. Life 8(3): 223-246

Hornby G S, Lipson H and Pollack J B 2001a Evolution of generative design systems for modular physical robots 4 : 4146-4151

Hornby G S, Pollack J B and et al 2001b Body-brain co-evolution using l-systems as a generative encoding. In: Proceedings of the Genetic and Evolutionary Computation Conference (GECCO-2001), pp. 868-875

Hornby G S, Lipson H and Pollack J B 2003 Generative representations for the automated design of modular physical robots. Robot. Autom., IEEE Trans. 19(4): 703-719

Jeong I-K and Lee J-J 1997 Evolving cooperative mobile robots using a modified genetic algorithm. Robot. Autonomous Syst. 21(2): 197-205

Kalra P and Prakash N R 2003 A neuro-genetic algorithm approach for solving the inverse kinematics of robotic manipulators. In: Systems, Man and Cybernetics, 2003. IEEE International Conference on, vol. 2, pp. 1979-1984

Klaus G, Glette K and Høvi M 2013 Evolving locomotion for a 12-dof quadruped robot in simulated environments. Biosystems 112(2): 102-106

Koos S, Mouret J-B and Doncieux S 2010 Crossing the reality gap in evolutionary robotics by promoting transferable controllers. In: Proceedings of the 12th Annual Conference on Genetic and Evolutionary Computation, pp. 119-126. ACM

Koos S, Mouret J-B and Doncieux S 2013 The transferability approach: Crossing the reality gap in evolutionary robotics. Evolut. Comput., IEEE Trans. 17(1): 122-145

Kuehn T J 2012 Evolutionary fabrication: a system of autonomous invention. In: Proceedings of the Fourteenth International Conference on Genetic and Evolutionary Computation Conference Companion, pp. 579-584. ACM

Lee W P, Hallam J and Lund H H 1996 A hybrid gp/ga approach for co-evolving controllers and robot bodies to achieve fitness-specified tasks. In: Evolutionary Computation, 1996, Proceedings of IEEE International Conference on, pp. 384-389

Lindenmayer A 1968 Mathematical models for cellular interactions in development i. filaments with onesided inputs. J. Theoret. Biol. 18(3): 280-299

Lipson H 2005 Evolutionary robotics and open-ended design automation. Biomimetics 17(9): 129-155

Lipson H and Pollack J B 2000 Automatic design and manufacture of robotic lifeforms. Nature 406(6799): 974-978

Lipson H and Pollack J 2006 Evolving physical creatures. In: Artificial Life VII: Proceedings of the seventh international conference on artificial life, pp. 282-287

Lund H H, Hallam J and Lee W-P 1997 Evolving robot morphology. In: Evolutionary Computation, 1997, IEEE International Conference on, pp. 197-202

McCulloch W S and Pitts W 1943 A logical calculus of the ideas immanent in nervous activity. Bull. Math. Biophys. 5(4): 115-133

Meeden L A 1996 An incremental approach to developing intelligent neural network controllers for robots. Syst. Man Cybern., B: Cybern., IEEE Trans. 26(3): 474-485

Michalewicz Z 1996 Genetic algorithms +data structures=evolution programs. Springer

Montes-Gonzalez F and Contreras C M 2013 The evolution of motivated and modulated robot selection. Int. J. Adv. Robot. Syst. 10

Morse G, Risi S, Snyder C R and Stanley K O 2013 Single-unit pattern generators for quadruped locomotion. In: Proceeding of the Fifteenth Annual Conference on Genetic and Evolutionary Computation Conference, pp. 719-726. ACM 
Nelson A L, Grant E, Galeotti J M and Rhody S 2004 Maze exploration behaviors using an integrated evolutionary robotics environment. Robot. Autonom. Syst. 46(3): 159-173

Nolfi S and Parisi D 1995 Evolving non-trivial behaviors on real robots: an autonomous robot that picks up objects. In: Topics in Artificial Intelligence, pp. 243-254. Springer

Nordin P and Banzhaf W 1997 An on-line method to evolve behavior and to control a miniature robot in real time with genetic programming. Adaptive Behav. 5(2): 107-140

Paul C and Bongard J C 2001 The road less travelled: Morphology in the optimization of biped robot locomotion. In: Intelligent Robots and Systems, 2001. Proceedings. 2001 IEEE/RSJ International Conference on, vol. 1, pp. 226-232

Pfeifer J C B R 2002 A method for isolating morphological effects on evolved behaviour. In: From Animals to Animats, 7: Proceedings of the Seventh International Conference on Simulation of Adaptive Behavior, volume 7, pp. 305. MIT Press

Piltan F, Sulaiman N, Jalali A and Aslansefat K 2011a Evolutionary design of mathematical tunable fpga based mimo fuzzy estimator sliding mode based lyapunov algorithm: Applied to robot manipulator. Int. J. Robot. Autom. 2(5): 317-343

Piltan F, Sulaiman N, Roosta S, Gavahian A and Soltani S 2011b Evolutionary design of backstepping artificial sliding mode based position algorithm: Applied to robot manipulator. Int. J. Eng. 5(5): 419-434

Piltan F, Yarmahmoudi M, Mirzaie M, Emamzadeh S and Hivand Z 2013 Design novel fuzzy robust feedback linearization control with application to robot manipulator. Int. J. Intell. Syst. Appl. (IJISA) 5(5): 1

Pires E S, Machado J T and de Moura Oliveira P B 2004 Robot trajectory planning using multi-objective genetic algorithm optimization. In: Genetic and Evolutionary Computation-GECCO 2004, pp. 615-626. Springer

Pollack J B and Lipson H 2000 The golem project: Evolving hardware bodies and brains. In: Evolvable Hardware, 2000. Proceedings. The Second NASA/DoD Workshop on, pp. 37-42

Pollack J B, Lipson H, Ficici S, Funes P, Hornby G and Watson R A 2000 Evolutionary techniques in physical robotics. In: Evolvable Systems: from biology to hardware, pp. 175-186

Pollack J B, Lipson H, Hornby G and Funes P 2001 Three generations of automatically designed robots. Artif. Life 7(3): 215-223

Pratihar D K 2003 Evolutionary roboticsa review. Sadhana 28(6): 999-1009

Pratihar D K 2013 Soft computing: fundamentals and applications. Ltd: Alpha Science International

Pratihar D K, Deb K and Ghosh A 1999 A genetic-fuzzy approach for mobile robot navigation among moving obstacles. Int. J. Approx. Reason. 20(2): 145-172

Ram A, Boone G, Arkin R and Pearce M 1994 Using genetic algorithms to learn reactive control parameters for autonomous robotic navigation. Adaptive Behav 2(3): 277-305

Rieffel J and Sayles D 2010 Evofab: A fully embodied evolutionary fabricator. In: Evolvable Systems: From Biology to Hardware, pp. 372-380. Springer

Risi S and Stanley K O 2013 Confronting the challenge of learning a flexible neural controller for a diversity of morphologies. In: Proceeding of the Fifteenth Annual Conference on Genetic and Evolutionary Computation Conference, pp. 255-262. ACM

Rout B and Mittal R 2010 Optimal design of manipulator parameter using evolutionary optimization techniques. Robotica 28(3): 381-395

Rubrecht S, Singla E, Padois V, Bidaud P and De Broissia M 2011 Evolutionary design of a robotic manipulator for a highly constrained environment. In: New Horizons in Evolutionary Robotics, pp. 109-121. Springer

Saad M S, Jamaluddin H and Darus I Z M 2012 Implementation of pid controller tuning using differential evolution and genetic algorithms. Int. J. Innov. Comput. Inf. Control 8(11): 7761-7779

Shenfield A and Fleming P J 2014 Multi-objective evolutionary design of robust controllers on the grid. Eng. Appl. Artif. Intell. 27: 17-27

Shukla A, Singla E, Wahi P and Dasgupta B 2013 A direct variational method for planning monotonically optimal paths for redundant manipulators in constrained workspaces. Robotics Autonom. Syst. 61(2): 209-220 
Sims K 1994a Evolving 3d morphology and behavior by competition. Artif. life 1(4): 353-372

Sims K 1994b Evolving virtual creatures. In: Proceedings of the 21st Annual Conference on Computer Graphics and Interactive Techniques, pp. 15-22. ACM

Singla E, Tripathi S, Rakesh V and Dasgupta B 2010 Dimensional synthesis of kinematically redundant serial manipulators for cluttered environments. Robotics Autonom. Syst. 58(5): 585-595

Smith T 1997 Adding vision to khepera: An autonomous robot footballer. Master's thesis, School of Cognitive and Computing Sciences, University of Sussex

Stanley K O and Miikkulainen R 2002 Evolving neural networks through augmenting topologies. Evolution. Comput. 10(2): 99-127

Tolley M T, Hiller J D and Lipson H 2011 Evolutionary design and assembly planning for stochastic modular robots. In: New Horizons in Evolutionary Robotics, pp. 211-225. Springer

Wiener-Ehrlich W K, Bart W M and Millward R 1980 An analysis of generative representation systems. J. Math. Psychol. 21(3): 219-246

Zykov V, Mytilinaios E, Desnoyer M and Lipson H 2007 Evolved and designed self-reproducing modular robotics. IEEE Trans. Robot. 23(2): 308-319 